\title{
Crystalline Oxide Solid Solutions in Oxygen Potential Gradients
}

\author{
H. Schmalzried, W. Laqua, and P. L. Lin \\ Institut für physikalische Chemie, Technische Universität Hannover, and SFB 126
}

Z. Naturforsch. 34a, 192-199 (1979); received October 27, 1978

\begin{abstract}
The steady state demixing of an initially homogeneous oxide solid solution (A, B)O in an oxygen potential field is studied theoretically and experimentally.

In case that $D_{\mathrm{A}}>D_{\mathrm{B}} \gg D_{0}$, the crystal is shifted with respect to the oxide lattice system toward the higher oxygen potential and is enriched in $A$ at the side of the higher oxygen potential, while the transport of oxygen in the crystal is negligible. A numerical solution of the transport problem is presented, and the predicted effect is verified experimentally.
\end{abstract}

\section{Introduction}

If homogeneous multicomponent phases are brought under the influence of a gradient of generalized thermodynamic potentials, demixing occurs normally. In this paper a new demixing effect of this type will be analyzed.

Firstly, the experimental fact shall be described: A homogeneous crystalline oxide solution (for example $(\mathrm{Co}, \mathrm{Mg}) \mathrm{O})$, the oxygen ion sublattice of which is practically immobile $\left(D_{\mathrm{Co}}, D_{\mathrm{Mg}} \gg D_{\mathrm{O}}\right)$, is brought between two compartments with different chemical potential of oxygen. Although the oxygen ions are immobile, after attaining steady state conditions it is found experimentally that a strong demixing process took place, resulting in an inhomogeneous oxide solid solution. $\mathrm{CoO}$ is enriched at the high oxygen potential side. In order to understand this process, it is expedient to discuss the situation that occurs if not a solid solution $(\mathrm{Co}, \mathrm{Mg}) \mathrm{O}$, but a $\mathrm{CoO}$-single crystal is placed in an oxygen potential gradient. In this case it was shown [1] that a cation vacancy concentration gradient occurs which sets up a vacancy flux from the side of the high oxygen potential to the side of the low oxygen potential. Since $\mathrm{CoO}$ is essentially a p-type semiconductor [2], as a consequence of the cation vacancy flux cobalt ions along with compensating electronic defects are transported towards the crystal surface that is in contact with the higher oxygen potential. This then has led to a macroscopic shift of the CoO-crystal in that direction in spite of the immobility of the oxygen ions. Details on the corresponding experiments can be found in [1]. It may be noted in passing that the moving interface between crystal and gas at the side of the

Reprint requests to Prof. Dr. H. Schmalzried, Callinstraße 3-3A, D-3000 Hannover 1.

$0340-4811 / 79 / 0200-0192 \$ 01.00 / 0$ low oxygen potential has been found unstable. The process can be described as an analogue of an oxydation reaction of metal A with constant reaction layer thickness; hence the linear rate of advancement of the interfaces.

The case of solid solutions (A, B)O in an oxygen potential gradient is consequently related to an alloy oxidation reaction. In contrast to the tarnishing reaction, however, again the reaction layer thickness remains approximately constant, and the mathematics is somewhat easier to handle than in case of the tarnishing reaction where the thickness grows parabolically. It has been attempted in the case of alloy oxidation to obtain quantitative results for the concentration distribution of cations A and B in the parabolically growing oxide layer $[3,4]$. In view of the foregoing, the problem to be theoretically solved and experimentally verified can be formulated as follows: Given a single crystalline solid solution (A, B)O between two different oxygen potentials, what is the steady state distribution of the cations if the oxygen ions are immobile, and what is the steady state velocity of the interfaces relative to a convenient frame of reference.

It may be worthwhile to point out the practical aspects of this problem: In almost all situations where multicomponent oxides are used at high temperatures as construction materials, oxygen potential gradients are present (e.g. in steelmaking) that cause a demixing of the initially homogeneous solid. This can severely alter the physical and (electro-)chemical properties of the construction material.

If the transport coefficients of the cations $A$ and $\mathrm{B}$ are given as functions of the mole fraction $X_{\mathrm{A}}$ in $(\mathrm{A}, \mathrm{B}) \mathrm{O}$, the transport problem can be solved as is shown in the next section. The self-(tracer-) 
diffusion coefficients $D_{i}$ of cations in simple transition metal oxide solid solutions were found to vary with $X_{\mathrm{A}}$ and $p_{\mathrm{O}_{2}}$ as [5]:

$$
D_{i}=D_{i}^{0} p_{\mathrm{O}_{2}}^{1 / n} \exp \left(b X_{\mathrm{A}}\right) ; \quad i=\mathrm{A}, \mathrm{B} .
$$

$n$ and $b$ are constants to be determined in tracer diffusion experiments. Hence

$$
D_{\mathrm{A}} / D_{\mathrm{B}}=D_{\mathrm{A}} 0 / D_{\mathrm{B}}^{0}=\beta^{0} .
$$

Normally, however, correlation effects of the elementary vacancy jumps occur that make $\beta^{0}$ (slightly) dependent on $X_{\mathrm{A}}$, although the vacancy concentration itself conforms to an equation that is the analogue of Equation (1). Correlation effects are found if the elementary jump frequency $\Gamma_{\mathrm{A}}$ of an A-cation into a neighbouring vacancy differs from $\Gamma_{\mathrm{B}}$. The effective jump frequencies that determine the cation mobility and diffusivity differ from $\Gamma$ by a correlation factor which is concentration dependent [6]. It is in principle possible to obtain additional information on correlation effects in oxide solid solutions by a comparison of calculated demixing profiles (including correlation effects) with experimentally determined steady state concentration profiles.

\section{Formal Treatment of the Transport Problem}

It is assumed that the molar volume is constant and that the oxide solid solution is semiconducting in the composition range covered by the demixing process. It is furthermore assumed that local thermodynamic equilibrium prevails in analogy to the oxidation of metals theory [7]. Neglecting as usual the cross terms in the transport coefficient matrix for crystal compounds one has in the laboratory (oxygen lattice) system:

$$
j_{\mathrm{A}}=-\frac{D_{\mathrm{A}} \cdot c_{\mathrm{A}}}{R T} \frac{\partial \eta_{\mathrm{A}^{2+}}}{\partial \xi}
$$

where $\eta_{\mathrm{A}^{2+}}$ is the electrochemical potential $\left(=\mu_{\mathrm{A}^{2+}}+2 F \varphi\right)$ of the ions $\mathrm{A}^{2+}$ (in the oxides considered $\gtrsim 99.9 \%$ of the metal atoms is doubly ionized). A corresponding equation holds for $j_{\mathrm{B}}$. The interaction between ideally diluted individual vacancies is assumed to be neglegible. If the (immobile) oxygen ions are predetermining the fixed number of regular cation sites, the vacancy flux $j_{\mathrm{v}}$ is given as

$$
j_{\mathrm{v}}=-\left(j_{\mathrm{A}}+j_{\mathrm{B}}\right) \text {. }
$$

For electroneutrality sake the vacancy flux induces an equivalent flux of electrons or electron holes, as is shown schematically in Figure 1. If one formulates the flux of electron holes in analogy to Eq. (3), one has

$$
j_{\mathrm{h}} \cdot=-\frac{D_{\mathrm{h}} \cdot c_{\mathrm{h}} \cdot}{R T} \frac{\partial \eta_{\mathrm{h}}}{\partial \xi} .
$$

Inserting Eqs. (3) and (5) into Eq. (4), assuming $2 j_{\mathrm{v}^{\prime \prime}}=j_{\mathrm{h}} \cdot$ (which means that doubly ionized cation vacancies do exist) and taking into account the semiconducting property of the solid solution ( $\left.D_{\text {ion }} c_{\text {ion }} \ll D_{\mathrm{h}} \cdot c_{\mathrm{h}} \cdot\right)$, one finds immediately that

$$
\mathrm{d} \mu_{\mathrm{h}} \cong-F \mathrm{~d} \varphi
$$

which, by virtue of the equations $\mu_{\mathrm{A}}+2 \mu_{\mathrm{h}}{ }^{*}=\mu_{\mathrm{A}^{2+}}$, $\mu_{\mathrm{B}}+2 \mu_{\mathrm{h}} \cdot=\mu_{\mathrm{B}^{2+}}$ and the local equilibrium assumption, yields in combination with Eq. (3)

$$
j_{\mathrm{A}}=-\frac{D_{\mathrm{A}} c_{\mathrm{A}}}{R T} \frac{\partial \mu_{\mathrm{A}}}{\partial \xi}
$$

and a corresponding equation for $j_{\mathrm{B}} \cdot \mu_{\mathrm{A}}$ and $\mu_{\mathrm{B}}$ are the chemical potentials of metal $A$ and $B$ in neutral form.

In addition, due to the assumption of local equilibrium, one can formulate in view of the reactions $\mathrm{A}+\mathrm{O}=\mathrm{AO}, \mathrm{B}+\mathrm{O}=\mathrm{BO}$ :

$$
\mu_{\mathrm{A}}+\mu_{\mathrm{O}}=\mu_{\mathrm{AO}} ; \mu_{\mathrm{B}}+\mu_{\mathrm{O}}=\mu_{\mathrm{BO}} .
$$

Since $(A, B) O$ is supposed to be an ideal solution with respect to the $\mathrm{AO}$ and $\mathrm{BO}$ components, and using the definition $\mu_{\mathrm{M}}=\mu_{\mathrm{M}^{0}}+R T \ln X_{\mathrm{M}}$, it is found from Eqs. (7) and (8) that

$$
\begin{aligned}
& j_{\mathrm{A}}=-\frac{D_{\mathrm{A}} X_{\mathrm{A}}}{V_{\mathrm{m}}}\left[\frac{\partial \ln X_{\mathrm{A}}}{\partial \xi}-\frac{\partial}{\partial \xi}\left(\frac{\mu_{0}}{R T}\right)\right], \\
& j_{\mathrm{B}}=-\frac{D_{\mathrm{B}} X_{\mathrm{B}}}{V_{\mathrm{m}}}\left[\frac{\partial \ln X_{\mathrm{B}}}{\partial \xi}-\frac{\partial}{\partial \xi}\left(\frac{\mu_{0}}{R T}\right)\right] .
\end{aligned}
$$

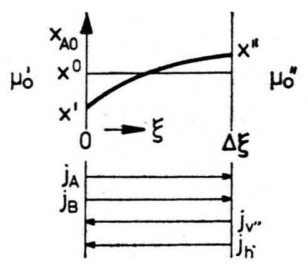

Fig. 1. Schematic representation of the AO-concentration $\left(X_{\mathrm{AO}}\right)$ and the vacancy- and particle fluxes $\left(V^{\prime \prime}=\right.$ cation vacancy, $h \cdot=$ electron hole) in an (A, B)O-solid solution with $D_{\mathrm{A}}>D_{\mathrm{B}}$ under the action of an oxygen potential field with $\mu_{0}^{\prime \prime}>\mu_{0}^{\prime}$. 
Equations (9) are the cationic flux equations in terms of the relevant variables of the transport problem. In case of non-ideal solid solutions $X_{M}$ in Eqs. (9) has to be replaced by $X_{M} f_{M}, f_{M}$ being the activity coefficient.

The total derivative of the concentration is

$$
\frac{\mathrm{d} c_{i}}{\mathrm{~d} t}=\frac{\partial c_{i}}{\partial t}+v \cdot \frac{\partial c_{i}}{\partial \xi},
$$

$v$ being the local velocity of a volume element relative to the lattice system (laboratory system). Since $\partial c_{i} / \partial t=-\operatorname{div} j_{i}$, one has from Eq. (10), after attaining steady state conditions:

$$
\partial / \partial \xi\left(j_{i}-v c_{i}\right)=0 \text {. }
$$

If one takes into account Eq. (4) and notes that $v=-V_{\mathrm{m}} \cdot j_{\mathrm{v}}=V_{\mathrm{m}}\left(j_{\mathrm{A}}+j_{\mathrm{B}}\right)$, it is found from the integrated form of Eq. (11) that

$$
v=\frac{\mathrm{d} \xi^{0}}{\mathrm{~d} t}=\frac{j_{\mathrm{A}}}{c_{\mathrm{A}}}=\frac{j_{\mathrm{B}}}{c_{\mathrm{B}}} .
$$

Equation (12) is the steady state condition, and $\xi^{0}$ is the locus of the crystal surface. Inserting Eq. (12) into (9) and eliminating $v$, one obtains

$$
\mathrm{d}\left(\frac{\mu_{0}}{R T}\right)=\frac{\gamma-X_{\mathrm{A}}}{X_{\mathrm{A}}\left(1-X_{\mathrm{A}}\right)} \cdot \mathrm{d} X_{\mathrm{A}} .
$$

Equation (13) relates the compositional change $\mathrm{d} X_{\mathrm{A}}=-\mathrm{d} X_{\mathrm{B}}$ to the change of the local oxygen potential in the solid solution. $\gamma$ is $D_{\mathrm{A}} / D_{\mathrm{A}}-D_{\mathrm{B}}$. In case that $D_{\mathrm{A}}$ and $D_{\mathrm{B}}$ are given by Eq. (1), $\gamma=\gamma^{0}=D_{\mathrm{A}}^{0} / D_{\mathrm{A}}{ }^{0}-D_{\mathrm{B}}{ }^{0}$ is independent of $X_{\mathrm{A}}$ or $\mu_{0}$.

On integration of (13) it is found that

$$
\begin{aligned}
\mu_{0} & -\mu_{0}\left(\xi^{0}\right) \\
& =R T\left[\gamma_{0} \ln \frac{X_{\mathrm{A}}(\xi)}{X_{\mathrm{A}}\left(\xi^{0}\right)}-\left(1-\gamma^{0}\right) \ln \frac{1-X_{\mathrm{A}}(\xi)}{1-X_{\mathrm{A}}\left(\xi^{0}\right)}\right]
\end{aligned}
$$

which, by setting $\xi=\xi^{\prime}, \xi^{0}=\xi^{\prime \prime}$ (' and " marking the two crystal surfaces) and $\Delta \mu_{0}=\mu_{0}{ }^{\prime \prime}-\mu_{0}^{\prime}$, yields

$$
\frac{\left(X_{\mathrm{A}^{\prime \prime}}\right)^{\gamma^{0}}}{\left(1-X_{\mathrm{A}}{ }^{\prime \prime}\right)^{\gamma^{0}-1}}=\exp \left\{\frac{\Delta \mu_{0}}{R T}\right\} \frac{\left(X_{\mathrm{A}}^{\prime}\right)^{\gamma^{0}}}{\left(1-X_{\mathrm{A}}\right)^{\gamma^{0}-1}} .
$$

With the abbreviation

$$
G\left(X_{\mathrm{A}}, \frac{\Delta \mu_{0}}{R T}\right)=\exp \left\{\frac{\Delta \mu_{0}}{R T}\right\} \frac{\left(X_{\mathrm{A}}\right)^{\gamma^{0}}}{\left(1-X_{\mathrm{A}}\right)^{\gamma^{0}-1}}
$$

one obtains

$$
G\left(X_{\mathrm{A}}{ }^{\prime \prime}, 0\right)=G\left(X_{\mathrm{A}^{\prime}}, \Delta \mu_{0} / R T\right) .
$$

The function $G\left(X_{\mathrm{A}}, \Delta \mu_{0} / R T\right)$ has been plotted in Fig. 2 with $\gamma^{0}$ equal to 1.322 , the value as found experimentally for $D_{\mathrm{A}} / D_{\mathrm{A}}-D_{\mathrm{B}}$ in $(\mathrm{Co}, \mathrm{Mg}) \mathrm{O}$ [6]. The solution of Eq. (15) in terms of $X_{\mathrm{A}^{\prime}}$ and $X_{\mathrm{A}}{ }^{\prime \prime}$ can be read from Fig. 2 as shown schematically in Figure 3 b.

In order to obtain numerical values for both $X_{\mathrm{A}}{ }^{\prime}$ and $X_{\mathrm{A}}{ }^{\prime \prime}$ at given $\Delta \mu_{0}$, a second equation in addition to Eq. (15) is needed. It is provided by the mass balance, which reads

$X_{\mathrm{A}^{0}} \cdot \Delta \xi=\int_{0}^{\Delta \xi} X_{\mathrm{A}} \cdot \mathrm{d} \xi=X_{\mathrm{A}}{ }^{\prime \prime} \cdot \Delta \xi-\int_{X_{\mathrm{A}^{\prime}}}^{X_{\mathrm{A}^{\prime \prime}}} \xi \cdot \mathrm{d} X_{\mathrm{A}}$

where $X_{\mathrm{A}^{0}}$ is the initial concentration of the homogeneous solid solution and $\Delta \xi=\xi^{\prime \prime}-\xi^{\prime}$ (see Figure 1). Equation (16) can be rewritten in the following form:

$$
X_{\mathrm{A}} 0=X_{\mathrm{A}}^{\prime \prime}-\int_{X_{\mathrm{A}^{\prime}}}^{X_{\mathrm{A}^{\prime \prime}}}(\xi \mid \Delta \xi) \cdot \mathrm{d} X_{\mathrm{A}}
$$

$(\xi / \Delta \xi)$ can be derived as a function of $X_{\mathrm{A}}$ as follows: From Equations (9), (12) and (13) one finds

$$
v=\gamma^{0} D_{\mathrm{B}}^{0} f\left(X_{\mathrm{A}}, \mu_{0}\right) \cdot \frac{1}{X_{\mathrm{A}}\left(1-X_{\mathrm{A}}\right)} \frac{\mathrm{d} X_{\mathrm{A}}}{\mathrm{d} \xi}
$$

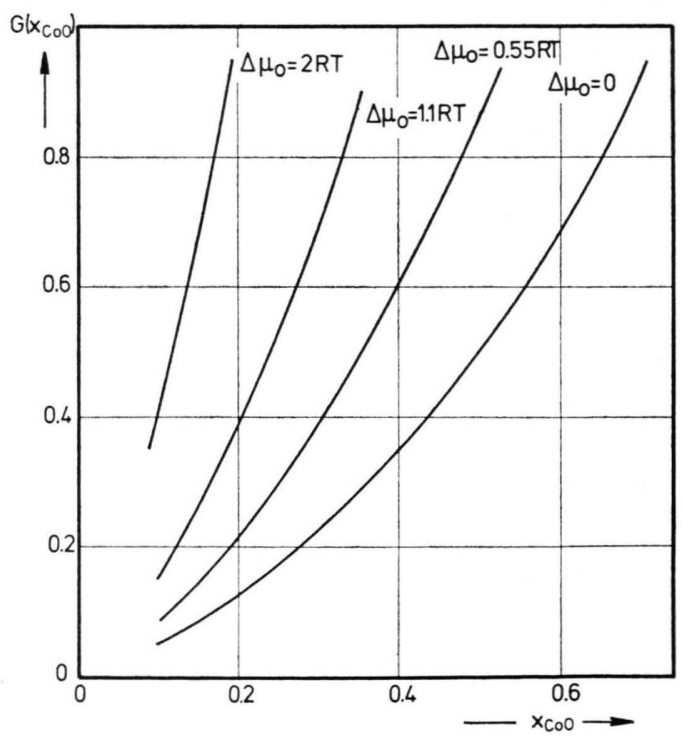

Fig. 2. Plots of the function $G\left(X_{\mathrm{CoO}}, \Delta \mu_{0}\right)$. The values $\Delta \mu_{0}=0.55 R T$ and $\Delta \mu_{0}=1.1 R T$ correspond to the experimental conditions with $p_{\mathrm{O}_{2}}^{\prime \prime} / p_{\mathrm{O}_{2}}^{\prime}=3.0$ and 9.2 , respectively. 
where $f\left(X_{\mathrm{A}}, \mu_{0}\right)=p_{\mathrm{O}_{2}}^{1 / n} \cdot \exp \left(b X_{\mathrm{A}}\right)$. Integration of Eq. (17) yields

$$
v=\frac{\gamma^{0} D_{\mathrm{B}^{0}}}{\xi\left(X_{\mathrm{A}}\right)} \cdot \int_{X_{\mathrm{A}^{\prime}}}^{X_{\mathrm{A}}} \frac{f\left(X_{\mathrm{A}}, \mu_{0}\right)}{X_{\mathrm{A}}\left(1-X_{\mathrm{A}}\right)} \mathrm{d} X_{\mathrm{A}} .
$$

Therefore, under steady state conditions:

$$
\frac{\xi\left(X_{\mathrm{A}}\right)}{\Delta \xi}=\frac{\int_{X_{\mathrm{A}^{\prime}}}^{X_{\mathrm{A}}} \frac{f\left(X_{\mathrm{A}}, \mu_{0}\right)}{X_{\mathrm{A}}\left(1-X_{\mathrm{A}}\right)} \cdot \mathrm{d} X_{\mathrm{A}}}{\int_{X_{\mathrm{A}^{\prime}}}^{X_{\mathrm{A}^{\prime \prime}}} \frac{f\left(X_{\mathrm{A}}, \mu_{0}\right)}{X_{\mathrm{A}}\left(1-X_{\mathrm{A}}\right)} \cdot \mathrm{d} X_{\mathrm{A}}} .
$$

Equation (18) can be introduced into Eq. (16a) in order to provide the relation for the determination of both $X_{\mathbf{A}^{\prime}}$ and $X_{\mathrm{A}}{ }^{\prime \prime}$. After some rearrangement, Eqs. (18) and (16a) yield:

$$
\begin{aligned}
\int \frac{f\left(X_{\mathrm{A}}, \mu_{0}\right)}{1-X_{\mathrm{A}}} & \mathrm{d} X_{\mathrm{A}}-X_{\mathrm{A}}{ }^{0} \\
& \times \int_{?}^{\prime \prime} \frac{f\left(X_{\mathrm{A}}, \mu_{0}\right)}{X_{\mathrm{A}}\left(1-X_{\mathrm{A}}\right)} \mathrm{d} X_{\mathrm{A}}=0 .
\end{aligned}
$$

In order to solve Eqs. (15) and (19) for $X_{\mathrm{A}^{\prime}}$ and $X_{\mathrm{A}^{\prime}}{ }^{\prime \prime}$, the following procedure is recommended: Let us define

$$
\begin{aligned}
F\left(X_{\mathbf{A}}, X_{\mathrm{A}}{ }^{0}, k\right)= & \int_{k}^{X_{\mathrm{A}}} \frac{f\left(X_{\mathrm{A}}, \mu_{0}\right)}{1-X_{\mathrm{A}}} \mathrm{d} X_{\mathrm{A}}-X_{\mathrm{A}}{ }^{0} \\
& \cdot \int_{k}^{X_{\mathrm{A}}} \frac{f\left(X_{\mathrm{A}}, \mu_{0}\right)}{X_{\mathrm{A}}\left(1-X_{\mathrm{A}}\right)} \cdot \mathrm{d} X_{\mathrm{A}},(20)
\end{aligned}
$$

$k$ being a constant value $0<k<X_{\mathrm{A}^{\prime}}$. Equation (19) can then also be written as

$$
F\left(X_{\mathbf{A}^{\prime}}, X_{\mathrm{A}^{0}}, k\right)=F\left(X_{\mathbf{A}^{\prime \prime}}, X_{\mathbf{A}^{0}}, k\right) .
$$

A schematic plot of $F\left(X_{\mathrm{A}}, X_{\mathrm{A}}{ }^{0}, k\right)$ is shown in Figure 3a. The graphical solution of Eqs. (15) and (20) simultaneously is also shown in Figure 3 . It is necessary to find those values of $X_{\mathrm{A}}{ }^{\prime}$ and $X_{\mathrm{A}}{ }^{\prime \prime}$ that satisfy both Eqs. (15) and (20). Equation (15a) has been given in Figure 2. In order to $\operatorname{plot} F\left(X_{\mathrm{A}}, X_{\mathrm{A}^{0}}, k\right)$, the following auxiliary integrals are introduced:

$$
\begin{aligned}
I\left(X_{\mathrm{A}}\right)= & \int_{0.05}^{X_{\mathrm{A}}} \exp \left(b X_{\mathrm{A}}\right) \\
& \cdot\left[X_{\mathrm{A}}^{\left(\gamma^{0}-\lambda-1\right) /(\lambda+1)} /\left(1-X_{\mathrm{A}}\right)^{\left(\gamma^{0}+\lambda\right) /(\lambda+1)}\right] \mathrm{d} X_{\mathrm{A}},
\end{aligned}
$$

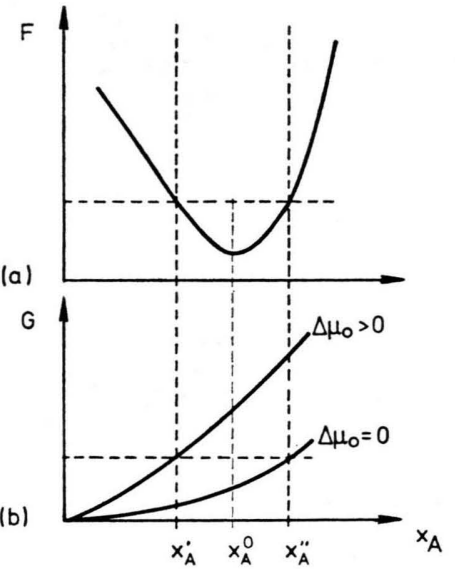

Fig. 3. Schematic plot of functions $F\left(X_{A}, X_{\AA}^{\circ}, k\right)$ and $G\left(X_{\mathrm{A}}, \Delta \mu_{0}\right)$, illustrating the graphical solution of Eqs. (13) and (19).

$$
\begin{aligned}
\tilde{I}\left(X_{\mathrm{A}}\right)=\int_{0.05}^{X_{\mathrm{A}}} \exp \left(b X_{\mathrm{A}}\right) \\
\cdot\left[X_{\mathrm{A}}^{\gamma^{0} /(\lambda+1)} /\left(1-X_{\mathrm{A}}\right)^{\left(\gamma^{0}+\lambda\right) /(1+\lambda)}\right] \mathrm{d} X_{\mathrm{A}}
\end{aligned}
$$

$I\left(X_{\mathrm{A}}\right)$ and $\tilde{I}\left(X_{\mathrm{A}}\right)$ may be tabulated. If one omitts a constant factor that cancels in Eqs. (19) or (21), $F\left(X_{\mathrm{A}}, X_{\mathrm{A}}^{0}, k\right)$ is then to be written as

$$
F\left(X_{\mathrm{A}}, X_{\mathrm{A}}{ }^{0}, k\right)=\tilde{I}\left(X_{\mathrm{A}}\right)-X_{\mathrm{A}^{0}} I\left(X_{\mathrm{A}}\right) \text {. }
$$

In introducing Eqs. (22) and (23), use has been made of writing $f\left(X_{\mathbf{A}}, \mu_{0}\right)$ in the following form:

$f\left(X_{\mathrm{A}}, \mu_{0}\right)=\exp \left(b X_{\mathrm{A}}\right) \cdot \exp \frac{\mu_{0}-\mu_{0} 0}{(\lambda+1) R T}$.

Equation (25) is equivalent to the previously given term $f\left(X_{\mathrm{A}}, \mu_{0}\right)=\exp \left(b X_{\mathrm{A}}\right) p_{\mathrm{O}_{2}}^{1 / n}$ as found experimentally. However, in the form of Eq. (25) it is possible to apply easily the well known principles of point defect thermodynamics [8, 9]. A geometrical representation of Eq. (20) is given in Figure 4.

\section{Experimental Procedure}

Single crystal slices $\left(\sim 500 \mu \mathrm{m}\right.$ thickness, $\left.\sim 1 \mathrm{~cm}^{2}\right)$ were cleaved from $\mathrm{MgO}$ (4 N quality, W. C. Spicer, England). They were reacted at $1470^{\circ} \mathrm{C}$ with $\mathrm{CoO}$ powder in alumina crucibles to form (Co, $\mathrm{Mg}) \mathrm{O}$ solid solutions by interdiffusion. In order to obtain crystals with an average mole fraction $X_{\mathrm{CoO}} \approx 0.5$, the annealing time was normally 3 weeks. These inhomogeneous ( $\mathrm{Co}, \mathrm{Mg}) \mathrm{O}$-slices were mechanically cleaned from adherent CoO-powder and precision- 


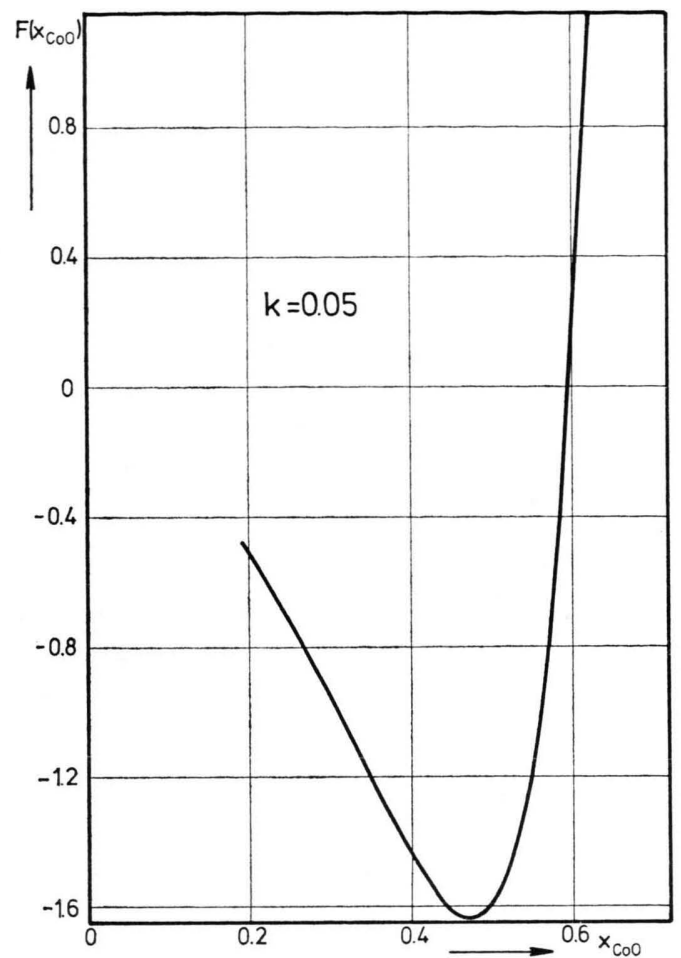

Fig. 4. Plot of the function $F\left(X_{\mathrm{CoO}}, X_{\mathrm{CoO}}^{0}, k\right)$ for $b=7.50$, $\gamma_{0}=1.322$ (both extrapolated from [6]) and $X_{\mathrm{CoO}}^{0}=0.47$, which is the initial homogeneous concentration of $\mathrm{CoO}$ in the solid solution shown in Figure 6a (2).

ground in order to produce parallel surfaces. A subsequent homogenization anneal was done in alumina crucibles partly filled with a $(\mathrm{Co}, \mathrm{Mg}) \mathrm{O}$ powder, the composition of which was approximately that of the corresponding single crystal. In this way evaporation losses were avoided. The crystal was suspended inside the crucible using a thin Pt-wire. Annealing times of 12 weeks at $1480^{\circ} \mathrm{C}$ were required to achieve homogenization, which was checked with the help of an electron microprobe. The final polish was done with a $3 \mu \mathrm{m}$ diamond wheel.

The set-up is shown schematically in Figure 5. Essentially, a small gas-chamber of $\sim 0.5 \mathrm{~cm}$ depth was ground into a two-bore alumina tube. The inner surface of this chamber was then reacted with $\mathrm{CoO}$ to form $\mathrm{CoAl}_{2} \mathrm{O}_{4}$. This spinel coating is necessary to avoid again losses of $\mathrm{CoO}$ from the single crystalline $(\mathrm{Co}, \mathrm{Mg}) \mathrm{O}$-solid solution during the demixing anneal in the oxygen potential field. The (Co, Mg)O-single crystal was joined to the polished alumina tube by means of platinum paste and gaskets under some firm pressure at $1470^{\circ} \mathrm{C}$. Two additional polycrystalline ( $\mathrm{Co}, \mathrm{Mg}) \mathrm{O}$-buffer discs and a thin wavy platinum foil between them were mounted as shown during the joining procedure. The foil compensated small deviations from parallelity. After the joining anneal, the buffer discs were removed and the gas-tightness of the platinum joints was tested.

All diffusion runs were made at $1439 \pm 2{ }^{\circ} \mathrm{C}$. The temperature was controlled at the outer surface of the $(\mathrm{Co}, \mathrm{Mg}) \mathrm{O}$-sample by means of a $\mathrm{Pt} / \mathrm{Pt} 10 \mathrm{Rh}$ thermocouple. Also, the outer surface of the sample was exposed to streaming air, whereas a $\mathrm{N}_{2} / \mathrm{O}_{2}$-gas mixture was flushed along the inner surface through the bores of the alumina tube. The oxygen partial pressure was measured before and after the gas chamber with a conventional solid state galvanic cell using $\mathrm{CaO}$-stabilized zirconia as an electrolyte.

After attaining a steady state concentration profile in the sample upon annealing in the oxygen

(Co, Mg) O-

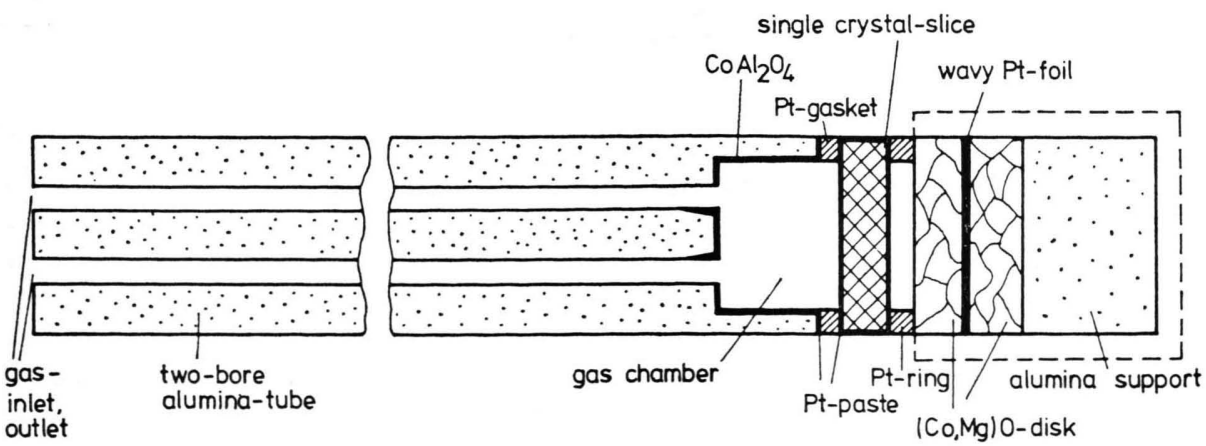

Fig. 5. Schematic representation of the experimental arrangement for placing a $(\mathrm{Co}, \mathrm{Mg}) \mathrm{O}$ single crystal in an oxygen potential field. The part surrounded with the dashed line is removed after the crystal has been joined to the aluminatube. 
potential field, the $(\mathrm{Co}, \mathrm{Mg}) \mathrm{O}$-crystal was cut from the tube join, mounted in plastic and sectioned parallel to the diffusion direction. After polishing, the sectioned samples were coated with a thin carbon film and the steady state concentration profile was determined with the electron microprobe (type EMX-SM07, ARL). X-ray intensities of Coand $\mathrm{Mg}-\mathrm{K}_{\alpha}$-lines were measured and converted to concentrations with the help of standard samples of $\mathrm{Co}_{x} \mathrm{Mg}_{1-x} \mathrm{O}$.

\section{Results and Discussion}

Two representative experimental concentration profiles of initially homogeneous $(\mathrm{Co}, \mathrm{Mg}) \mathrm{O}$-single crystals after attainment of the steady state in an oxygen potential field are shown in Fig. 6 a along with theoretical curves. Note that the experimental conditions in both cases are quite different. In full agreement with the theoretical deductions of Section 2, a strong demixing effect is found in the single crystalline ( $\mathrm{Co}, \mathrm{Mg}) \mathrm{O}$-samples, the higher Co-concentration is found at the higher oxygen potential side. The annealing time has been proven long enough to establish steady state conditions.
Since the area below the horizontal line of the initial concentration of $\mathrm{Co}$, and below the experimental concentration curve after demixing is nearly the same, it is concluded that evaporation losses are negligible. Although the overall agreement between experimental and calculated steady state concentration profiles is quite good, it is obvious that the experimental curve deviates to some extent from the theoretical profile at the lower oxygen potential side. An interpretation is given in the following.

A discussion of the atomistic transport processes for pure $\mathrm{CoO}$-single crystals in an oxygen potential gradient can be found in the literature [1]. In accordance with this discussion the steady state demixing is the result of a cation vacancy flux from the high oxygen potential surface to the low oxygen potential surface and the higher jump frequency of Co-ions into a neighbouring vacancy as compared with the jump frequency of Mg-ions [6]. The low potential interface, where the cation vacancy flux arrives to induce the reaction

$$
\mathrm{V}_{\text {cat. }}^{\prime \prime}+2 \mathrm{~h} \cdot+(\mathrm{MeO})_{\mathrm{s}}=1 / 2 \mathrm{O}_{2}(\mathrm{~g})+\mathrm{Me}_{\text {cat. }}^{2+}
$$

$(\mathrm{Me}=\mathrm{Co}, \mathrm{Mg}$; cat. denotes cation and s surface) is

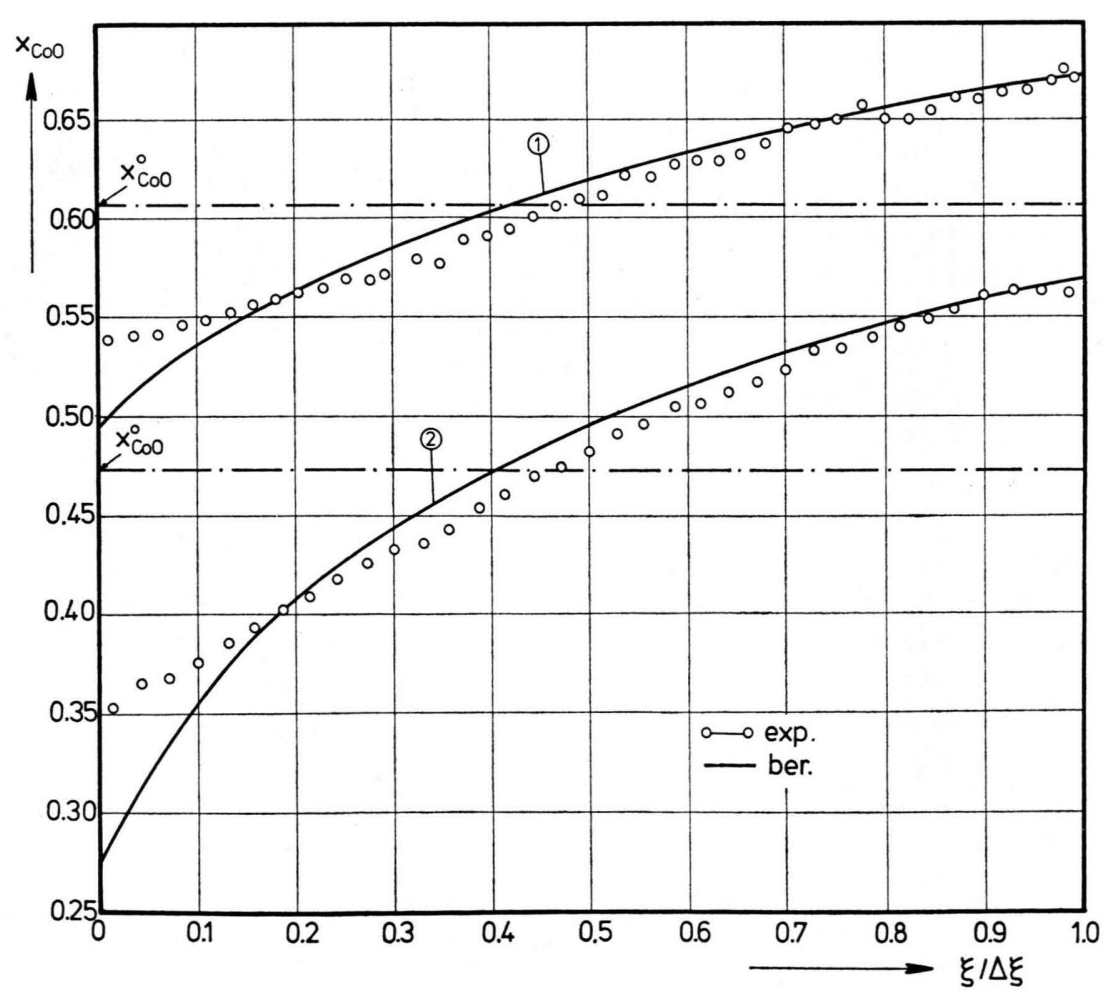

Fig. 6a. Experimental and calculated concentration profiles for (Co, $\mathrm{Mg}) \mathrm{O}$ solid solutions.

(1) $X_{\text {coo }}^{0}=0.61$, annealed for about $240 \mathrm{~h}$ at $1439^{\circ} \mathrm{C}$ in an oxygen potential field with $p_{\mathrm{O}_{2}}^{\prime \prime} / p_{\mathrm{O}_{2}}^{\prime}=$ 3.0.

(2) $X_{\mathrm{CoO}}^{0}=0.47$, annealed for about $240 \mathrm{~h}$ at $1438^{\circ} \mathrm{C}$ in an oxygen potential field with $p_{\mathrm{O}^{2}}^{\prime \prime} / p_{\mathrm{O}_{2}}^{\prime}=9.2$. 


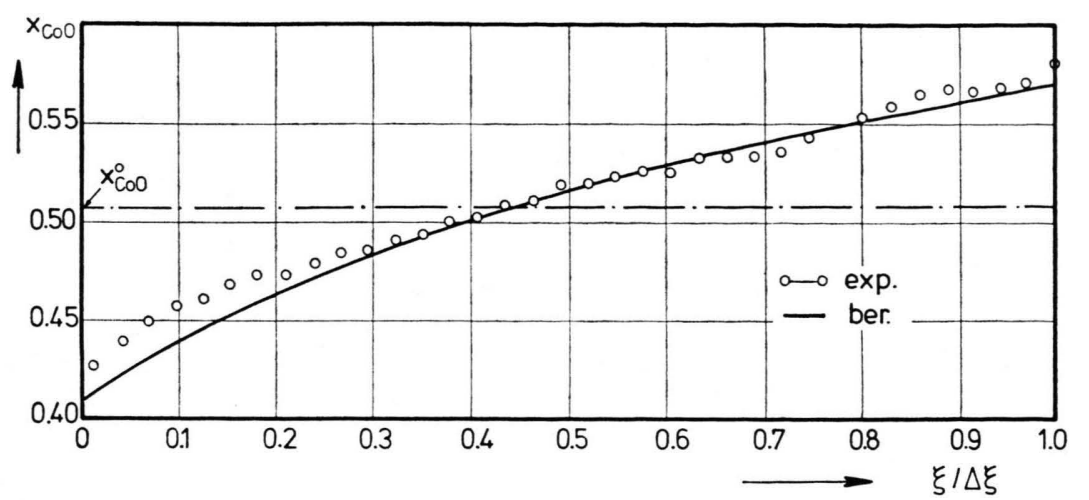

Fig. 6b. Experimental and calculated concentration profiles in analogy to Fig. 6a (1) but with a reduced annealing time. The experimental conditions are: $X_{\text {coo }}^{0}$ $=0.51, \quad t=126 \mathrm{~h}, \quad T=1439^{\circ} \mathrm{C}$ and $p_{\mathrm{O}_{2}}^{\prime \prime} / p_{\mathrm{O}_{2}}^{\prime}=3.0$. instable during its motion towards the high oxygen potential side, since any disturbance in form of an indentation of the plane surface with a corresponding higher vacancy gradient increases the flux of vacancies towards this disturbance, thus enhancing the indentation growth. This self-accelerating process results in a jagged low oxygen potential surface with pores which, after some time, can be found throughout the crystal between the highand the low oxygen potential side. Therefore, the oxygen potential gradient in the heavily pore-filled region of the crystal is smaller than the theoretical oxygen potential gradient as calculated from the original crystal geometry. Consequently, the vacaney flux in this region is lowered and the extent of demixing is smaller than is to be expected from theory. This is exactly what is found in Figure 6 a. Photographs of the unstable interface in case of a $\mathrm{CoO}$-single crystal in an oxygen potential gradient can be found in [1]; they resemble very much the situation as found in this investigation with $(\mathrm{Co}, \mathrm{Mg}) \mathrm{O}$-solid solutions.

It is also the instability of the gas/solid interface and the pore formation that does not allow to experimentally verify the steady state velocity of the moving interface of the crystal in an oxygen potential gradient, since a quantitative assessment of the influence of the pore volume on the interface velocity $\mathrm{v}$ is not possible. The theoretical values of $\mathbf{v}$ according to Eq. (17) agree within a factor of 2 with the experimental ones, the theoretical values being the smaller ones as should be expected.

Clearly, the influence of pore formation on the steady state concentration curves is smallest in the early stages of the crystal displacement provided that steady state conditions are already established. This is why demixing runs were made with short annealing times. In this case the low oxygen potential interface is still unjagged and the single crystalline solution compact with the exception of a small number of isolated pores. Figure $6 \mathrm{~b}$ shows the demixing profile after $126 \mathrm{~h}$ of annealing in contrast to the results of the $240 \mathrm{~h}$ experiment shown in Figure 6a. There is still a slight discrepancy between experiment and theory, which may eventually stem from micropores or the nonattainment of the steady state in this interface region.

\section{Conclusions}

The action of generalized thermodynamic potential gradients upon homogeneous solid solutions results in demixing. Application of the correct transport equations and boundary plus steady state conditions results in a theoretical description of the demixing process that can be verified experimentally. For the sake of illustration the experiments were done with $(\mathrm{Co}, \mathrm{Mg}) \mathrm{O}$-solid solutions since the transport properties of this materials have been studied extensively. While the oxygen transport in these crystals is negligible, a strong demixing effect is found with the cations if the sample is exposed to an oxygen potential gradient. Since this effect occurs quite often in practical situations with high temperature materials, the calculations as presented here may contribute to control the materials deterioation.

\section{Acknowledgement}

We are indebted to the Deutsche Forschungsgemeinschaft for financial support. 
[1] G. J. Yurek and H. Schmalzried, Ber. Bunsenges. physik. Chem. 79, 255 (1975).

[2] R. Dieckmann, Z. physik. Chem. N.F. 107, 189 (1977).

[3] C. Wagner, Corr. Sci. 9, 91 (1969).

[4] B. D. Bastow, D. P. Whittle, and G. C. Wood, Proc. Roy. Soc. London 356, 177 (1977).

[5] G. Schwier, R. Dieckmann, and H. Schmalzried, Ber. Bunsenges. physik. Chem. 77, 402 (1973).
[6] R. Dieckmann and H. Schmalzried, ibid. 79, 1108 (1975).

[7] C. Wagner, Z. physik. Chem. B 21, 25 (1933).

[8] F. A. Kröger, The Chemistry of Imperfect Crystals North-Holland Publ. Comp., Amsterdam 1964.

[9] H. Schmalzried and A. Navrotsky, Festkörperthermodynamik, Verlag Chemie GmbH, Weinheim 1975. 